\title{
The Antiangiogenic Therapy in Ovarian Cancer
}

\author{
Loizzi Vera*1, Cicinelli Ettore ${ }^{1}$, Del Vecchio Vittoria ${ }^{1}$, Naglieri Emanuele ${ }^{2}$, Ranieri Girolamo ${ }^{3}$ and Cormio \\ Gennaro ${ }^{1,2}$
}

${ }^{1}$ Department of Biomedical Sciences and Human Oncology, University of Bari, Italy

${ }^{2}$ Gynecologic Oncology Unit, IRCCS Istituto Tumori Giovanni Paolo II, Bari, Italy

${ }^{3}$ Interventional and Medical Oncology Unit, IRCCS Istituto, Tumori Giovanni Paolo II, Bari, Italy

Received: May 21, 2018; Published: May 29, 2018

*Corresponding author: Vera Loizzi, Department of Biomedical Sciences and Human Oncology, University of Bari, Italy, Piazza Giulio Cesare 11, Italy

Keywords: Angiogenesis; Vegf; Ovarian Cancer

\section{Mini Review}

The standard treatment for epithelial ovarian cancer (EOC) consists of optimal cytoreductive surgery followed by platinum-based chemotherapy [1-2]. Bevacizumab has been approved for the treatment of several tumors such as colorectal cancer, non-squamous non-small cell lung cancer, renal cell carcinoma, epithelial ovarian, fallopian tube, and primary peritoneal cancers. The use of antiangiogenic agent in EOC is based on the results of randomized clinical trials which revealed an improved survival rates with the addition of bevacizumab to standard first-line chemotherapeutic drugs [34]. In addition, this benefit has been evaluated in second-line setting both in platinum sensitive and platinum-resistant disease [56]. Although standard treatment for EOC is optimal cytoreductive surgery followed by platinum-based chemotherapy [7-8], the role of interval debulking surgery (IDS) after a short course of neoadjuvant chemotherapy (NACT) has also studied and represents an alternative in women unable to undergo upfront complete resection. In fact, no differences in progression-free survival (PFS) were evaluated in EOC patients undergoing IDS compared to those treated with primary debulking surgery. However, few adverse effects and lower mortality rates were observed in the group which included patients treated with IDS [9-10].

\section{Neo Adjuvant Setting}

Bevacizumab in the neoadjuvant setting have been studied in few studies [11-12] with controversial results. Therefore its use is not yet recommended in this setting because based on wound complications, gastrointestinal perforations and fistulas, and thromboembolic events [13]. The first study that studied the efficacy and toxicity of preoperative chemotherapy with or without bevacizumab has been the NOVA study. It was a randomized, phase II, multicentric clinical trial. Patients with EOC were randomized to receive 4 cycles of chemotherapy based on carboplatin and paclitaxel with or without bevacizumab. After chemotherapy the patients underwent cytoreductive surgery and postoperative bevacizumab for a total of 22 cycles. No significant response rate was observed even if a higher rate of optimal surgeries in the bevacizumab group was evaluated [12]. The confirmation of the role of bevacizumab in this setting will arrive from an other study. In fact the ANTHALYA study is an ongoing, randomized, open-label, phase II trial involving patients with initially unresectable advanced stage ovarian, tubal, or peritoneal carcinoma [14]. Another controversial point is the appropriate interval between bevacizumab administration and subsequent surgery in order to reduce the risk of impaired wound healing/wound dehiscence. There is a consensus to undergo surgery for at least 28 days following their last treatment [15].

\section{First Line Setting}

The efficacy of bevacizumab in addition to the standard for firstline treatment based on carboplatin and paclitaxel was showed in two randomized multicenter trials [3-4]. The GOG-218 trial [3] was conducted in patients affected by advanced stage epithelial ovarian, fallopian tube, or peritoneal cancer submitted to first-line chemotherapeutic. The study showed a significantly increase in progression free survival (PFS) in patients receiving bevacizumab plus standard chemotherapy followed by maintenance bevacizumab with respect to patients treated only with standard chemotherapy or without bevacizumab maintenance (14.1 v/s 10.3 and 11.2 months, respectively, $\mathrm{p}<0.001)$. However, these results were not similar for the overall survival (OS), therefore the addition of bevacizumab to standard chemotherapy did not significantly improve the OS. However, a further analysis in patients with only stage IV disease demonstrated improvement in the median OS; it was significantly higher in the group submitted to maintenance bevacizumab versus no maintenance or no bevacizumab. ICON7 trial was the sec- 
ond phase III randomized trial that showed similar results [4]. An improved in median PFS was observed in the bevacizumab group ( $p=0.0041$ ). The overall response rate was $48 \%$ in the chemotherapy-alone group and $67 \%$ in the bevacizumab group, respectively ( $\mathrm{p}<0.0001$ ). However, similarly to the other study, no differences in the OS rate were observed. Only for patients with FIGO stage III sub-optimally debulked or stage IV disease or who had not undergone debulking surgery an OS increase of 9 months was showed. (p $=0.03)[15]$.

\section{Recurrent OC Setting}

Platinum-sensitive OC woman is defined who is having a progression-free interval $\geq 6$ months from the last platinum chemotherapy. Patients who relapse within this interval are usually treated with platinum-based therapy consisting in combination of carboplatin with paclitaxel or pegylated liposomal doxorubicin (PLD), or gemcitabine [16-18]. Two studies (OCEANS and GOG-213 trials) have studied the role of bevacizumab in the platinum-sensitive recurrent OC setting. The OCEANS is a randomized phase III trial with patients assigned to the standard chemotherapy (intravenous gemcitabine plus carboplatin) with or without bevacizumab $(15 \mathrm{mg} / \mathrm{kg})$ until disease progression or unacceptable toxicity. The results showed an imporved PFS in the bevacizumab group than in the placebo group $(\mathrm{p}<0.001)$ [5]. In addition, the overall response rate was higher in the bevacizumab group than in the controls $(p<0.0001)$. However, no improvement in OS was observed. Toxicity in terms of hypertension was higher in the bevacizumab group. For these reasons, bevacizumab has been approved in combination with carboplatin and gemcitabine for patients with a first recurrence of platinum-sensitive EOC or fallopian tube or primary peritoneal cancer who has not received prior therapy with VEGF agents. The second trial is the Gynecologic Oncology Group (GOG)-0213 which has been conducted in patients affected by platinum-sensitive EOC or fallopian tube or peritoneal cancer. Data evaluated an increase in PFS $(p<0.0001)$ and in OS (p = 0.056) [6].

Platinum-resistant OC woman is defined who is having a progression-free interval $<6$ months from the last platinum chemotherapy. Several new cytotoxic drugs have been studied in chemoresistant EOC setting, such as bevacizumab. However, these patients have a poor outcome with a lower tumor response rates. Sehouli et al. have demonstrated that single-agent chemotherapy has been generally recommended based on the increased toxicity with combined therapy without a survival advantage [19]. The only randomized phase III multicenter trial evaluating the efficacy and tolerability of adding bevacizumab to single-agent chemotherapy in patients with recurrent, platinum-resistant epithelial ovarian, fallopian tube, or primary peritoneal cancer is the AURELIA study $[20,21]$. Patients received single-agent chemotherapy (paclitaxel, PLD, or topotecan) alone or in combination with bevacizumab until disease progression or unacceptable toxicity. The primary endpoint was PFS. The results showed that the median PFS was longer in patients receiving bevacizumab plus chemotherapy. However, no differences in OS were observed. However, to evaluate a definitive profile of efficacy and tolerability of bevacizumab in this setting of patients, the results of ongoing trials investigating bevacizumab alone or in combination with conventional antiblastic agents are necessary.

\section{References}

1. Bristow RE, Tomacruz RS, Armstrong DK (2002) Survival effect of maximal cytoreductive surgery for advanced ovarian carcinoma during the platinum era: a meta-analysis. J Clin Oncol 20(5): 1248-1259.

2. Piver MS, Lele SB, Marchetti DL (1988) The impact of aggressive debulking surgery and cisplatin-based chemotherapy on progressionfree survival in stage III and IV ovarian carcinoma. J Clin Oncol 6(6): 983-989.

3. Burger RA, Brady MF, Bookman MA (2011) Incorporation of bevacizumab in the primary treatment of ovarian cancer. N Engl J Med 365(26): 24732483.

4. Perren TJ, Swart AM, P fisterer J (2011) A phase 3 trial of bevacizumab in ovarian cancer. N Engl J Med 365(26): 2484-2496.

5. Aghajanian C, Blank SV, Goff BA (2012) OCEANS: a randomized, doubleblind, placebo-Controlled phase III trial of chemotherapy with or without bevacizumab in patients with platinum-sensitive recurrent epithelial ovarian, primary peritoneal, or fallopian tube cancer. J Clin Oncol 30(17): 2039-2045.

6. Coleman RL, Brady MF, Herzog TJ (2015) A phase III randomized controlled clinical trialof carboplatin and paclitaxel alone or in combination with bevacizumab followed by bevacizumab and secondary cytoreductive surgery in platinum-sensitive, recurrent ovarian, peritoneal primary and fallopian tube cancer (Gynecologic Oncology Group 213). Soc Gynecol Oncol Annu Meet Womens Cancer, Chicago, USA.

7. Bristow RE, Tomacruz RS, Armstrong DK (2002) Survival effect of maximal cytoreductive surgery for advanced ovarian carcinoma during the platinum era: a meta-analysis. J Clin Oncol 20(5): 1248-1259.

8. Piver MS, Lele SB, Marchetti DL (1988) The impact of aggressive debulking surgery and cisplatin-based chemotherapy on progressionfree survival in stage III and IV ovarian carcinoma. J Clin Oncol 6(6): 983-989.

9. Van Der Burg M, Van Lent M, Buyse M (1995) The effect of debulking surgery after induction chemotherapy on the prognosis in advanced epithelial ovarian cancer. Gynecological Cancer Cooperative Group of the European Organization for Research and Treatment of Cancer. N Engl J Med 332(10): 629-634.

10. Rose P, Nerenstone S, Brady M (2004) Secondary surgical cytoreduction for advanced ovarian carcinoma. N Engl J Med 351: 2489-2497.

11. Chereau E, Lambaudie E, Houvenaeghel G (2013) Morbidity of surgery after neoadjuvant chemotherapy including bevacizumab for advanced ovarian cancer. Int J GynecolCancer 23(7): 1326-1330.

12. Garcia Y, De Juan A, Mendiola C (2015) Phase II randomized trial of neoadjuvant (NA) chemotherapy(CT) with or without bevacizumab (Bev) in advanced epithelial ovarian cancer(EOC) (GEICO 1205/NOVA TRIAL) J Clin Oncol: 33.

13. Han E, Monk B (2007) What is the risk of bowel perforation associated with bevacizumab therapy in ovarian cancer? Gynecol Oncol 105(1): 3-6.

14. Rouzier R (2014) A randomized, open-label, phase II study assessing the efficacy and the safety of bevacizumab in neoadjuvant therapy in patients with FIGO stage IIIc/IV ovarian, tubal, or peritoneal adenocarcinoma, initially unresectable. J Clin Onc 32(15) TPS5614.

15. Oza AM, Perren TJ, Swart AM ICON7 (2013) final overall survival results in the GCIG phase III randomized trial of bevacizumab in women with newly diagnosed ovarian cancer. Eur Cancer Congr, Amsterdam. 
16. Bolis G, Scarfone G, Giardina G (2001) Carboplatin alone vs carboplatin plus epidoxorubicinas second-line therapy for cisplatin- orcarboplatinsensitive ovarian cancer. Gynecol Oncol 81(1): 3-9.

17. Parmar M, Ledermann J, Colombo N (2003) Paclitaxel plus platinumbased chemotherapy versus conventional platinum-based chemotherapy in women with relapsed ovarian cancer: the ICON4/AGO-OVAR-2.2 trial. Lancet 361(9375): 2099-2106.

18. P Fisterer J, Plante M, Vergote I (2006 ) Gemcitabine plus carboplatin compared with carboplatin

19. in patients with platinum-sensitive recurrent ovarian cancer: an intergroup trial of the AGO-OVAR, the NCIC CTG, and the EORTC GCG. J Clin Oncol 24(29): 4699-4707.

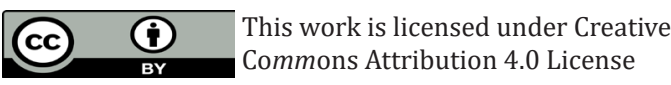

Submission Link: https://biomedres.us/submit-manuscript.php
20. Sehouli J, Stengel D, Oskay Oezcelik G (2008) Non platinum topotecan combinations versus topotecan alone for recurrent ovarian cancer: results of a phase III study of the North-Eastern German Society of Gynecological Oncology Ovarian Cancer Study Group. J ClinOncol 26(19): 3176-3182.

21. Pujade Lauraine E, Hilpert F, Weber B (2014) Bevacizumab combined with chemotherapy for platinum-resistant recurrent ovarian cancer: the AURELIA open-label randomized phase III trial. J ClinOncol 32(13): 1302-1308.

Assets of Publishing with us
- Global archiving of articles
RESEARCHES $\quad \begin{aligned} & \text { Immediate, unrestricted online access } \\ & \text { - Rigorous Peer Review Process }\end{aligned}$

